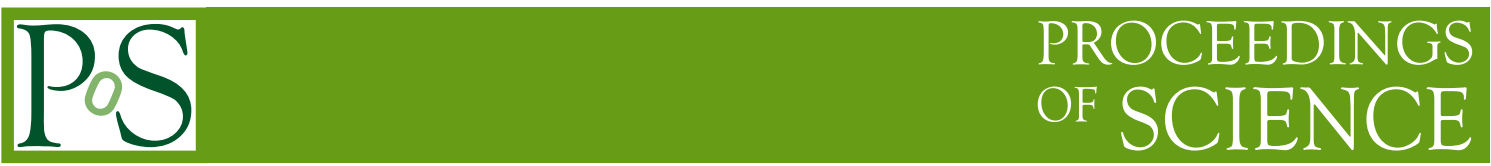

\title{
Exclusive dimuon measurements with LHCb
}

\author{
Tara SHEARS, on behalf of the LHCb collaboration. * \\ University of Liverpool \\ E-mail: tara.shearsecern.ch
}

\begin{abstract}
A measurement of exclusive dimuon production provides a clean test of QED and QCD predictions in a low multiplicity environment. Exclusively produced dimuon pairs can be produced by two photon fusion, or through resonances produced by pomeron-photon fusion or double pomeron exchange. We present cross-section measurements for exclusive dimuon production, and the first observations at a proton proton collider of exclusive $\mathrm{J} / \psi, \psi(2 S)$ and $\chi_{c}$ states, obtained with 37 $\mathrm{pb}^{-1}$ of data at a centre-of-mass energy of $7 \mathrm{TeV}$, collected by the LHCb experiment.
\end{abstract}

The 2011 Europhysics Conference on High Energy Physics-HEP 2011,

July 21-27, 2011

Grenoble, Rhônes Alpes France

${ }^{*}$ Speaker. 


\section{Introduction}

The process $p+p \rightarrow p+X+p$, where the protons remain intact and $\mathrm{X}$ is any final state, can proceed via two photon fusion, promeron-photon fusion or double pomeron exchange. A measurement of the precisely known QED two photon process can provide an indirect luminosity measurement. A measurement of the production cross-section of exclusively produced $\mathrm{J} / \psi, \psi(2 S)$ and $\chi_{c}$ states provides a test of QCD predictions in a low multiplicity environment.

The LHCb experiment is instrumented in the forward region with full tracking, calorimetry, and particle identification capability between pseudorapidities $\eta$ of $1.9 \leq \eta \leq 4.9$. Charged particle detection is provided within $-4.0 \leq \eta \leq-1.5$, by means of three planes of silicon detectors downstream of the interaction point. The experiment is capable of triggering dimuon pairs down to low transverse momentum $\left(p_{T}\right)$ and invariant mass $\left(M_{\mu \mu}\right)$ thresholds, whilst vetoing on additional detector activity. The measurements detailed here are made with a dataset of about $37 \mathrm{pb}^{-1}$.

\section{Event selection}

Candidate events must pass one of two low multiplicity triggers: either $M_{\mu \mu}>1 \mathrm{GeV}$ and $p_{T}(\mu \mu)>0.9 \mathrm{GeV}$, or $M_{\mu \mu}>2.7 \mathrm{GeV}$ with no requirement on muon $p_{T}$. An additional requirement is placed on the maximum hit multiplicity detected in the scintillating pad detector (placed in front of the electromagnetic calorimeter), to retain low multiplicity events. The offline event selection identifies muons (and photons), within the region $2.0 \leq \eta \leq 4.5$.

For the QED process, candidate events must have two muons which satisfy $M_{\mu \mu}<2.5 \mathrm{GeV}$, $p_{T}(\mu \mu)<0.1 \mathrm{GeV}$, and there must be no other tracks within the acceptance. Known resonances are removed by also cutting around the appropriate invariant mass. Backgrounds arise from inelastic production of dimuon pairs and double pomeron exchange, and are estimated directly from data. The background dimuon $p_{T}$ spectrum is obtained from events containing more than two tracks. This, together with a signal template obtained from LPAIR [1] simulation, are fitted to the distribution in data to extract the signal purity (97\%).

Exclusive $\mathrm{J} / \psi$ and $\psi(2 S)$ candidates are selected by requiring events to have two muons with $p_{T}(\mu \mu)<0.9 \mathrm{GeV}$, and dimuon invariant masses consistent with those expected for the relevant resonance. Candidates must have no other tracks within the acceptance. Backgrounds from inelastic production are estimated from data, and form approximately $30 \%$ of the event sample.

Exclusive $\chi_{c}$ candidates are selected by imposing the exclusive $\mathrm{J} / \psi$ selection, and requiring one photon in addition. The invariant mass of the two muons and photon must be consistent with the $\chi_{c}$ mass. Backgrounds from inelastic production (60\% of the sample), are estimated in the same way as before. The relative amounts of the $\chi_{c}^{0}: \chi_{c}^{1}: \chi_{c}^{2}$ states are estimated by scaling the expected masses for each to fit the distribution in data.

\section{Results}

The estimated number of signal events are corrected for detection and reconstruction efficiency, and divided by the effective integrated luminosity of the sample to yield the production cross-sections. More details of all of these procedures are given in [2]. 
All efficiencies are estimated from Monte Carlo. Systematic errors are estimated by comparing distributions in simulation and data, scaling to reflect any differences, and taking the difference with respect to the unscaled determination as the uncertainty.

Due to the restrictive multiplicity criteria in the event selection, only a fraction of the $37 \mathrm{pb}^{-1}$ with one proton proton interaction per crossing is used in the analysis. The effective integrated luminosity depends on the trigger efficiency and the average number of beam crossings. The total integrated luminosity is estimated from a combination of Van der Meer scan and beam gas collision data, and is subject to a $\pm 10 \%$ uncertainty for this dataset. The uncertainty on the effective integrated luminosity is estimated to be $20 \%$, and is the leading cause of uncertainty in the measurement.

Results are given in table 1, and compared to theoretical prediction. Predictions are known to within $1 \%$ for the QED process, but only to within about $20 \%$ for the QCD predictions. All results are consistent with predictions.

\begin{tabular}{|l|c|c|}
\hline Measurement & Data /pb & Theory /pb \\
\hline$\sigma(\mathrm{QED})$ & $67 \pm 10 \pm 7 \pm 15$ & $42[1]$ \\
$\sigma(J / \psi)$ & $474 \pm 12 \pm 51 \pm 92 \mathrm{pb}$ & $292[3], 330[4], 330[5], 710[6]$ \\
$\sigma(\psi(2 S))$ & $12.2 \pm 1.8 \pm 1.3 \pm 2.4 \mathrm{pb}$ & $6.1[3], 17[6]$ \\
$\sigma\left(\chi_{c}^{0}\right)$ & $9.3 \pm 2.2 \pm 3.5 \pm 1.8$ & $14[4]$ \\
$\sigma\left(\chi_{c}^{1}\right)$ & $16.4 \pm 5.3 \pm 5.8 \pm 3.2$ & $10[4]$ \\
$\sigma\left(\chi_{c}^{2}\right)$ & $28.0 \pm 5.4 \pm 9.7 \pm 5.4$ & $3[4]$ \\
\hline
\end{tabular}

Table 1: Measured exclusive dimuon cross-sections for the QED and QCD processes tested. For the data, the first error is statistical, the second systematic, and the third due to the luminosity estimate. Theory predictions are listed in the rightmost column, with relevant measurements carried out at other experiments.

\section{Conclusions}

Clear signals for exclusive $J / \psi, \psi(2 S)$ and $\chi_{c}$ mesons have been observed. The measured cross-sections for $J / \psi, \psi(2 S), \chi_{c}^{0}, \chi_{c}^{1}$ and $\chi_{c}^{2}$ are in agreement with the theoretical predictions which have large uncertainties. The measured cross-section for diphoton produced dimuons agrees with the theoretical estimate.

\section{References}

[1] J. Vermaseren, Nucl. Phys. B229, (1983) 347.

[2] The LHCb Collaboration, LHCb-CONF-2011-022.

[3] S. Klein and J. Nystrand, Phys. Rev. C60, (1999) 014903.

[4] L.A. Harland-Lang, V.A. Khoze, M.G. Ryskin, W.J. Stirling, arXiv:hep-ph/0909.4748.

[5] L. Motyka, G. Watt, Phys. Rev. D 78, (2008) 014023.

[6] W. Schafer, A. Szczurek, Phys.Rev. D76 (2007) 094014. arXiv:hep-ph/0811.2488 